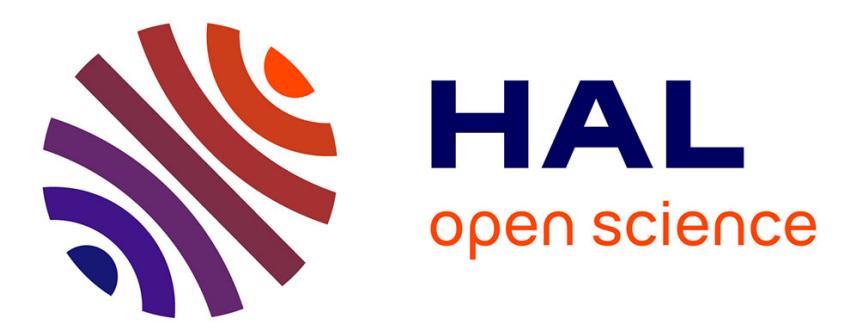

\title{
Numerically focused full-field swept-source optical coherence microscopy with low spatial coherence illumination
}

Anton Grebenyuk, Antoine Federici, Vladimir Ryabukho, Arnaud Dubois

\section{- To cite this version:}

Anton Grebenyuk, Antoine Federici, Vladimir Ryabukho, Arnaud Dubois. Numerically focused fullfield swept-source optical coherence microscopy with low spatial coherence illumination. Applied optics, 2014, 53 (8), pp.1697-1708. 10.1364/AO.53.001697 . hal-00956673

HAL Id: hal-00956673

https://hal-iogs.archives-ouvertes.fr/hal-00956673

Submitted on 7 Mar 2014

HAL is a multi-disciplinary open access archive for the deposit and dissemination of scientific research documents, whether they are published or not. The documents may come from teaching and research institutions in France or abroad, or from public or private research centers.
L'archive ouverte pluridisciplinaire HAL, est destinée au dépôt et à la diffusion de documents scientifiques de niveau recherche, publiés ou non, émanant des établissements d'enseignement et de recherche français ou étrangers, des laboratoires publics ou privés. 


\title{
Numerically focused full-field swept-source optical coherence microscopy with low spatial coherence illumination
}

\author{
Anton Grebenyuk, ${ }^{1, *}$ Antoine Federici, ${ }^{2}$ Vladimir Ryabukho, ${ }^{1,3}$ and Arnaud Dubois ${ }^{2}$ \\ 'Department of Optics and Biophotonics, Saratov State University, 83 Astrakhanskaya, Saratov 410012, Russia \\ 'Laboratoire Charles Fabry, Institut d'Optique Graduate School, CNRS UMR 8501, Université Paris-Sud, \\ 2 Avenue Augustin Fresnel, 91127 Palaiseau Cedex, France \\ ${ }^{3}$ Institute of Precision Mechanics and Control, Russian Academy of Sciences, 24 Rabochaya, \\ Saratov 410028, Russia \\ ${ }^{*}$ Corresponding author: grebenyukaa@yandex.ru
}

Received 28 May 2013; revised 9 December 2013; accepted 14 January 2014; posted 30 January 2014 (Doc. ID 191145); published 10 March 2014

\begin{abstract}
We propose a 3D imaging technique based on the combination of full-field swept-source optical coherence microscopy (FF-SSOCM) with low spatial coherence illumination and a special numerical processing that allows for numerically focused coherent-noise-free imaging without mechanical scanning in longitudinal or transversal directions. We show, both theoretically and experimentally, that the blurring effects arising in FF-SSOCM due to defocus can be corrected by appropriate numerical processing even when low spatial coherence illumination is used. A FF-SSOCM system was built for testing the performance of this technique. Coherent-noise-free imaging of a sample with longitudinal extent exceeding the optical depth of field is demonstrated without displacement of the sample or any optical element. (C) 2014 Optical Society of America

OCIS codes: (110.4500) Optical coherence tomography; (180.3170) Interference microscopy; (070.7345) Wave propagation; (100.3010) Image reconstruction techniques; (110.6880) Threedimensional image acquisition; (090.1995) Digital holography.

http://dx.doi.org/10.1364/AO.53.001697
\end{abstract}

\section{Introduction}

Low-coherence interferometry is a noncontact optical sensing technology that provides the possibility of 3D image reconstruction. In the most recent technique, referred to as optical coherence tomography (OCT), an optical probe directs a broadband light beam on the sample and sends reflected light back to the interferometer $[1,2]$. The interference pattern associated with each single scan point is interpreted as a depth profile (A-scan). In the time domain approach, the length of the interferometer reference arm is

$1559-128 \mathrm{X} / 14 / 081697-12 \$ 15.00 / 0$

(C) 2014 Optical Society of America scanned to bring forth the appearance of the interference signal. In the frequency domain approach, the reference arm length is fixed. The spectrum of the interference pattern is captured by the detector and then converted to the time domain using a Fourier transformation. Imaging speed is dramatically improved in the frequency domain OCT. In both approaches, a cross section (B-scan) is obtained by scanning the probe linearly across the sample. 3D images can be generated by combining multiple cross sections. Relatively low numerical aperture lenses are used in OCT to preserve a sufficient depth of field, especially in the frequency domain approach, where the depth profile (A-scan) is acquired in parallel. This limits the transverse resolution, which 
is a major drawback of OCT. Several solutions have been implemented to improve the transverse resolution, including dynamic focusing [3-5], illumination of the sample with a Bessel beam $[\underline{6}, \overline{7}]$, or image postprocessing as implemented, for example, in interferometric synthetic aperture microscopy (ISAM) [8].

Another way to achieve high transverse resolution imaging is to acquire en face images rather than cross sectional B-scan images using high numerical aperture optics, as can be accomplished in optical coherence microscopy (OCM). Two general approaches for OCM have been reported to date. The first approach of OCM is based on the combination of scanning confocal microscopy with low-coherence interferometry, as initially demonstrated in [9] and revisited later [10]. A drawback of this approach is the relatively slow acquisition speed, since three dimensions have to be scanned to get a 3D image. The second approach of OCM involves full-field illumination and detection using an area camera. En face sections are then acquired without scanning in the transversal directions. This approach has attracted a lot of attention because of its potentially faster operation speed for 3D imaging. It has been realized in a number of modalities in both the time domain and Fourier domain with spatially coherent illumination [11-13] and low spatial coherence illumination [14-17].

When achieved with plane-wave illumination (i.e., spatially coherent illumination), OCM corresponds to conventional digital holography or digital holographic microscopy (DHM) utilizing broadband or frequency tunable (swept) light sources. Like digital holography, these holographic OCM techniques offer the possibility of numerical refocusing without adjustment of the optical focus $[11-13,18,19]$. Therefore, when realized in the Fourier domain using frequency swept sources, these techniques can acquire $3 \mathrm{D}$ images without displacement of the sample or optical elements in either the transversal or longitudinal directions. This is very promising for highspeed 3D imaging. However, the transversal spatial coherence of plane-wave illumination makes these techniques very sensitive to coherent noise (e.g., because of dust in the optical path) and degrade image quality because of the possible presence of granular speckle and the manifestation of cross talk effects [20].

When achieved with partially spatially coherent (low coherence) illumination, the technique is usually termed as full-field optical coherence microscopy (FF-OCM), or sometimes full-field optical coherence tomography (FF-OCT). FF-OCM produces tomographic images in the en face orientation by the arithmetic combination of interferometric images acquired with an area camera and by illuminating the whole field to be imaged with low-coherence light (temporally and spatially). This technique has been developed mostly in the time domain with broadband low-coherence light sources, including light emitting diodes (LEDs) [14], thermal light lamps [16,17], or fluorescence-based sources [21]. Full-field OCM can image at high resolution with highly reduced coherent noise and cross talk effects [20]. However, as a time domain technique, it requires scanning of the longitudinal sample structures to produce a $3 \mathrm{D}$ image, which is time consuming.

Recently, a full-field OCT/OCM technique with transversally partially coherent illumination has been demonstrated in the frequency domain using a frequency swept source [22]. This work demonstrated that full-field swept-source OCM (FFSSOCM) provides the possibility of parallel detection of 3D image data with highly reduced coherent noise. Nevertheless, with this kind of technique, as in conventional frequency domain OCT, the imaged region is limited by the original depth of field related to the objective NA.

In this paper, we propose a 3D imaging technique, based on FF-SSOCM with partial spatial coherence illumination, in which the imaging depth is extended by numerical processing. This processing performs a numerical correction of the complex coherence signal in FF-SSOCM, including both correction of the angular spectrum gate (numerical focusing) and correction of the temporal spectrum gate (similarly to conventional Fourier domain OCT). This numerical processing takes into account the specific properties of illumination with an arbitrary degree of transversal spatial coherence. It thus allows for numerically focused coherent-noise-free 3D imaging without mechanical scanning in longitudinal or transversal directions. The theory of this numerical processing is developed based on a previous theoretical model [23,24]. A FF-SSOCM system was built for testing the performance of this numerical processing technique. Experimental observations confirm the theoretical predictions and the validity of the proposed method.

\section{Image Formation in FF-SSOCM}

\section{A. Interference Analysis}

The theoretical analysis presented in this paper is based on the theoretical model of image formation in optical microscopes proposed in [23,24]. This model is based on the scalar diffraction theory and combines an interference analysis similar to the Wiener-Khintchin theorem derivation of [25] with a special analysis of the optical microscope transmission functions.

To consider the signal delivered by a two-beam interference microscope using illumination with arbitrary transversal spatial coherence, we first need to analyze the interference of the optical fields from both arms of the interferometer. For this purpose some results from $[23,24]$ are summarized in this subsection.

The intensity of a quasi-monochromatic optical field of circular temporal frequency, $\omega$, in the registration plane $(x, y)$ can be considered proportional to the power spectral density of the field, $I(\omega ; x, y)$ : 


$$
\begin{aligned}
I(\omega ; x, y)= & I_{S}(\omega ; x, y)+I_{R}(\omega ; x, y)+\Gamma(\omega ; x, y) \\
& +\Gamma^{*}(\omega ; x, y),
\end{aligned}
$$

where the spectral densities, $I_{S}(\omega ; x, y)$ and $I_{R}(\omega ; x, y)$, are proportional to the intensities of the optical fields coming from the sample and the reference arms; the mutual spectral density, $\Gamma(\omega ; x, y)$, is proportional to their mutual coherence function. These functions are determined by the following expressions $[23,24]$ :

$$
\begin{aligned}
& 2 \pi \delta\left(\omega-\omega^{\prime}\right) I_{S}(\omega ; x, y)=\left\langle V_{S}(\omega ; x, y) V_{S}^{*}\left(\omega^{\prime} ; x, y\right)\right\rangle, \\
& 2 \pi \delta\left(\omega-\omega^{\prime}\right) I_{R}(\omega ; x, y)=\left\langle V_{R}(\omega ; x, y) V_{R}^{*}\left(\omega^{\prime} ; x, y\right)\right\rangle, \\
& 2 \pi \delta\left(\omega-\omega^{\prime}\right) \Gamma(\omega ; x, y)=\left\langle V_{S}(\omega ; x, y) V_{R}^{*}\left(\omega^{\prime} ; x, y\right)\right\rangle,
\end{aligned}
$$

where the functions, $V_{S}(\omega ; x, y)$ and $V_{R}(\omega ; x, y)$, are proportional to the complex amplitude distributions of the sample and reference fields. The angular brackets indifferently denote time averaging or statistical averaging, since the optical fields are supposed to be stationary.

To determine the averages in Eq. (2) we need to analyze the optical field propagation in the two interferometer arms from some initial planes $\left(x_{0}, y_{0}\right)$ to the registration plane $(x, y)$, i.e., determine the optical transmission functions of the two arms. The initial $\left(x_{0}, y_{0}\right)$ planes should be chosen in such a way that the optical field distributions in these planes are identical for both arms.

In the theoretical model $[23,24]$, the optical field propagation is analyzed using the Fresnel-Kirchhoff integral and angular spectrum representation. The imaging and illumination systems are treated under a parabolic approximation and with the assumption that the aberrations of the microscope optics are corrected for the analyzed part of the field of view in such a way that the general shape of the equations hold when higher numerical apertures are considered (some aberrations can still be taken into account by the aperture function, $A$ ). Such an assumption cannot be applied to the optical field propagation through the sample and thence it is treated more strictly for being applicable to high numerical aperture imaging (the ultimate limitation being the scalar approximation) [23].

Considering the back focal planes (aperture planes) of the objectives at the stage of illumination (before reflection from the sample and reference mirror) as the initial planes (Fig. 1), we can write for the reference field [24]:

$$
\begin{aligned}
V_{R}(\omega ; x, y) \approx & \mu(\omega ; x, y) \int \mathrm{d} x_{0} \mathrm{~d} y_{0} V_{0}\left(\omega ; x_{0}, y_{0}\right) \Phi_{R}^{2} \\
& \times\left(\omega ;-k \frac{x_{0}}{f^{\prime}},-k \frac{y_{0}}{f^{\prime}}\right) r_{R}\left(\omega ;-k \frac{x_{0}}{f^{\prime}},-k \frac{y_{0}}{f^{\prime}}\right) \\
& \times A\left(\omega ;-x_{0},-y_{0}\right) \exp \left[i k\left(x_{0} x+y_{0} y\right) / f_{L}^{\prime}\right],
\end{aligned}
$$

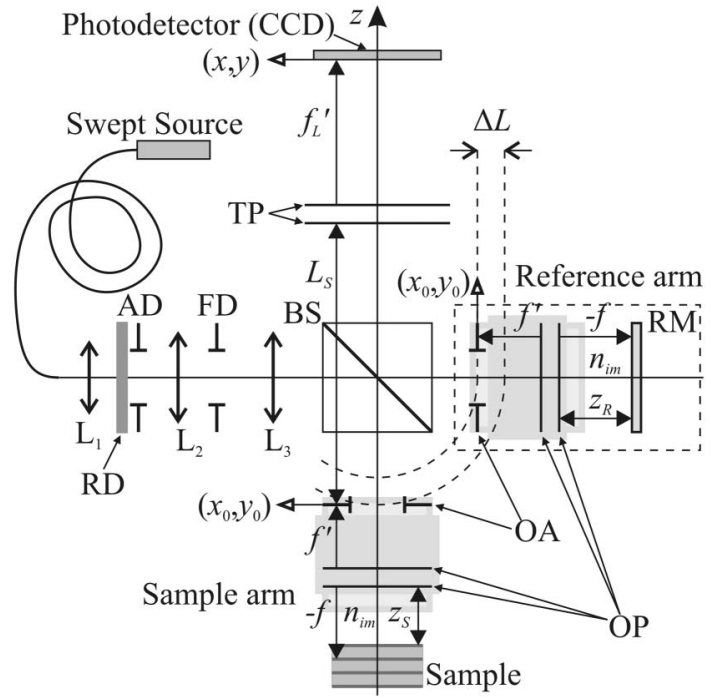

Fig. 1. Schematic of the FF-SSOCM setup. Imaging system: $\mathrm{OP}-$ principal planes of the microscope objectives; OA-aperture planes of the objectives; TP - principal planes of the tube lens; BS, cube beamsplitter; $(x, y)$, registration plane, where a matrix photodetector (CCD) is placed. Illumination system: light from the swept-source is guided via an optical fiber and directed by a lens system, $\mathrm{L} 1$, onto the rotating diffuser, $\mathrm{RD} ; \mathrm{AD}$, diaphragm of variable radius, located right after the diffuser and determining the illumination aperture size; lenses, L2 and L3, image AD onto the aperture planes, $\mathrm{OA} ; \mathrm{FD}$, field diaphragm of variable radius, which is optically conjugated by lens, L3, and the objectives' lenses with the front focal planes of the objectives.

$$
\begin{aligned}
\mu(\omega ; x, y)= & \frac{i}{\lambda f_{L}^{\prime}} \exp \left\{i k \left[2 f^{\prime}\left(1+n_{0}^{2}\right)+L\right.\right. \\
& \left.\left.+f_{L}^{\prime}\right]\right\} \exp \left[\frac{i \pi}{\lambda f_{L}^{\prime}}\left(1-\frac{L}{f_{L}^{\prime}}\right)\left(x^{2}+y^{2}\right)\right], \\
\Phi_{R}\left(\omega ; k_{x}, k_{y}\right) & =\exp \left[i\left(z_{R}-|f|\right)\left(k^{2} n_{0}^{2}-k_{x}^{2}-k_{y}^{2}\right)^{1 / 2}\right],
\end{aligned}
$$

where $f^{\prime}$ and $f_{L}^{\prime}$ are the back focal distances of the objective and tube lenses, respectively; $n_{0}=n_{\mathrm{im}}$ is the refractive index of immersion; $k=\omega / c$ is the wavenumber; $\lambda=2 \pi c / \omega$ is the wavelength; $r_{R}$ is the amplitude reflectivity of the reference mirror (which can depend on the reflection angle); $A\left(\omega ; x_{0}, y_{0}\right)$ is the aperture function of the objectives; $V_{0}\left(\omega ; x_{0}, y_{0}\right)$ is proportional to the complex amplitude distribution of the illuminating optical field immediately after passing the objective aperture plane; $L$ is the distance between the objective aperture plane (back focal plane) and the tube lens front principal plane; $z_{R}$ is the distance from the reference objective front principal plane to the reference mirror surface; $f$ is the front focal distance of the objectives. The function, $\Phi_{R}\left(\omega ; k_{x}, k_{y}\right)$, arises from the angular spectrum representation and describes the transformation of the angular spectrum due to propagation at a distance $z_{R}-|f| . \mu(\omega ; x, y)$ is a complex coefficient accounting mostly for the mean optical path of the field propagating through the microscope 
(interferometer arm) and its possible curvature due to the mismatch of the objective back focus and the tube lens front focus.

As a model object we shall consider an $N$-layered medium with layer thicknesses $\left\{\Delta z_{j}\right\}$ and refractive indices $\left\{n_{j}\right\}$. The upper $N$ interfaces have a uniform reflectivity, while the $(N+1)$ th interface has a transversal pattern, $r_{S}\left(\omega ; x_{S}, y_{S}\right)$, of the amplitude reflection coefficient distribution. Such a model object allows us to take into account the refractive properties of the immersion medium as well as the outer layers of the sample (and the respective coherence effects related to the refractive index variation [19,26-29]) and at the same time to avoid dealing with scattering of light by the outer layers (which is a usual simplification in OCT/OCM analysis of the defocus effects $[11,12,18,19,30])$ when analyzing the signal from the sample inner structure.

The function, $V_{S}(\omega ; x, y)$, can then be written in the following form $[\underline{24}, \underline{31}]$ :

$$
\begin{aligned}
& V_{S}(\omega ; x, y) \\
& \approx \frac{\mu(\omega ; x, y)}{\left(\lambda f^{\prime}\right)^{2}} \iint \mathrm{d} x_{S} \mathrm{~d} y_{S} r_{S}\left(\omega ; x_{S}, y_{S}\right) \\
& \quad \times \iint A\left(\omega ; x_{3}, y_{3}\right) T_{2}\left(\omega ; k \frac{x_{3}}{f^{\prime}}, k \frac{y_{3}}{f^{\prime}}\right) \\
& \quad \exp \left\{-i k\left[x_{3}\left(\frac{x}{f_{L}^{\prime}}+\frac{x_{S}}{f^{\prime}}\right)+y_{3}\left(\frac{y}{f_{L}^{\prime}}+\frac{y_{S}}{f^{\prime}}\right)\right]\right\} \mathrm{d} x_{3} \mathrm{~d} y_{3} \\
& \quad \times \iint V_{0}\left(\omega ; x_{0}, y_{0}\right) T_{1}\left(\omega ;-k \frac{x_{0}}{f^{\prime}},-k \frac{y_{0}}{f^{\prime}}\right) \\
& \quad \times \exp \left[-i k\left(x_{0} x_{S}+y_{0} y_{S}\right) / f^{\prime}\right] \mathrm{d} x_{0} \mathrm{~d} y_{0}, \\
& T_{1}\left(\omega ; k_{x}, k_{y}\right)=\prod_{j=1}^{N} t_{j-1, j}\left(\omega ; k_{x}, k_{y}\right) \\
& \prod_{j=0}^{N} \exp \left[i \Delta z_{j}\left(k^{2} n_{j}^{2}-k_{x}^{2}-k_{y}^{2}\right)^{1 / 2}\right], \\
& \prod_{j=0}^{N} \exp \left[i \Delta z_{j}\left(k^{2} n_{j}^{2}-k_{x}^{2}-k_{y}^{2}\right)^{1 / 2}\right],
\end{aligned}
$$

where $\Delta z_{0}=z_{S}-|f|, z_{S}$ being the distance from the sample arm objective front principal plane to the sample surface; $t_{j-1, j}$ and $t_{j, j-1}$ are the amplitude transmission coefficients from the $(j-1)$ th layer to the $j$ th layer and backward. The complex phase distributions in Eq. (6) arise from the angular spectrum representation of the optical field propagation through the sample, similarly to $\Phi_{R}$.

It is important to note that the fields arising due to the reflection from the $N$ upper interfaces are not taken into account here. $V_{S}(\omega ; x, y)$ represents only part of the actual optical field in the registration plane, corresponding solely to the reflection from the $(N+1)$ th interface in the sample. This is done because the interference between optical fields, generated by reflection from different interfaces, is usually considered as a spurious signal (apart from the autocorrelation low-coherence interferometry [32]) and is therefore not of interest in this paper. However, if necessary, these signals can as well be considered on the basis of this theory, by inclusion of corresponding fields in the $V_{S}(\omega ; x, y)$ function.

\section{B. Analysis of the Complex Coherence Function}

Let us assume the field $V_{0}\left(\omega ; x_{0}, y_{0}\right)$ in the illumination aperture plane $\left(x_{0}, y_{0}\right)$ to be spatially incoherent, which means that

$$
\begin{aligned}
& \left\langle V_{0}\left(\omega ; x_{0}, y_{0}\right) V_{0}^{*}\left(\omega^{\prime} ; x_{0}^{\prime}, y_{0}^{\prime}\right)\right\rangle \\
& \quad=2 \pi \delta\left(\omega-\omega^{\prime}\right) I_{0}\left(\omega ; x_{0}, y_{0}\right) \delta\left(x_{0}-x_{0}^{\prime}\right) \delta\left(y_{0}-y_{0}^{\prime}\right) .
\end{aligned}
$$

Substitution of Eqs. (3)-(7) into Eq. (2) allows for the analysis of the mutual coherence function, $\Gamma(\omega ; x, y)$, and of both the sample and reference intensities, $I_{S}(\omega ; x, y)$ and $I_{R}(\omega ; x, y)$.

Substitution of Eq. (3) into Eq. (2c) taking into account Eq. (7) yields:

$$
\begin{aligned}
\Gamma(\omega ; x, y) \approx & \mu_{0}(\omega) \iint \mathrm{d} x_{S} \mathrm{~d} y_{S} r_{S}\left(\omega ; x_{S}, y_{S}\right) \\
& \times \iint A_{2}\left(\omega ; x_{3}, y_{3}\right) \exp \left\{-i k\left[x_{3}\left(\frac{x}{f_{L}^{\prime}}+\frac{x_{S}}{f^{\prime}}\right)\right.\right. \\
& \left.\left.+y_{3}\left(\frac{y}{f_{L}^{\prime}}+\frac{y_{S}}{f^{\prime}}\right)\right]\right\} \mathrm{d} x_{3} \mathrm{~d} y_{3} \\
& \times \iint A_{1}\left(\omega ; x_{0}, y_{0}\right) \exp \left\{-i k\left[x_{0}\left(\frac{x}{f_{L}^{\prime}}+\frac{x_{S}}{f^{\prime}}\right)\right.\right. \\
& \left.\left.+y_{0}\left(\frac{y}{f_{L}^{\prime}}+\frac{y_{S}}{f^{\prime}}\right)\right]\right\} \mathrm{d} x_{0} \mathrm{~d} y_{0},
\end{aligned}
$$

where

$$
\mu_{0}(\omega)=\frac{|\mu(\omega ; x, y)|^{2}}{\left(\lambda f^{\prime}\right)^{2}}=\lambda^{-4} f^{\prime-2} f_{L}^{\prime-2},
$$

$$
\begin{aligned}
& A_{1}\left(\omega ; x_{0}, y_{0}\right) \\
& =I_{0}\left(\omega ; x_{0}, y_{0}\right) A^{*}\left(\omega ;-x_{0},-y_{0}\right) r_{R}^{*}\left(\omega ;-k \frac{x_{0}}{f^{\prime}},-k \frac{y_{0}}{f^{\prime}}\right) \\
& \quad \times T_{1}\left(\omega ;-k \frac{x_{0}}{f^{\prime}},-k \frac{y_{0}}{f^{\prime}}\right)\left[\Phi_{R}^{*}\left(\omega ;-k \frac{x_{0}}{f^{\prime}},-k \frac{y_{0}}{f^{\prime}}\right)\right]^{2}, \\
& A_{2}\left(\omega ; x_{3}, y_{3}\right)=A\left(\omega ; x_{3}, y_{3}\right) T_{2}\left(\omega ; k \frac{x_{3}}{f^{\prime}}, k \frac{y_{3}}{f^{\prime}}\right) .
\end{aligned}
$$

If we consider the situation when the objectives in the sample and reference arms are placed at different distances from the beam splitter (i.e., when the 
elements in the dashed box in Fig. 1 are shifted along the optical axis as a whole), then, strictly speaking, the optical fields in the aperture planes of the objectives are no longer identical and we have to select the initial plane earlier in the optical path. However, if this asymmetry, $\Delta L=L_{S}-L_{R}$, of the interference microscope arms is small compared to the longitudinal coherence length due to the angular spectrum of the illumination field in the objective aperture plane (a description of this type of coherence is given in $[33,34])$ and $\Delta L / \lambda \ll f_{L}^{\prime 2} /\left(x^{2}+y^{2}\right)_{\max }$, then the mutual coherence function can be approximated as:

$$
\Gamma_{L}(\omega ; x, y) \approx \exp [2 i k \Delta L] \Gamma(\omega ; x, y),
$$

where $\Gamma(\omega ; x, y)$ is determined by Eq. (8). Finally, it can be noted that, in the case when the approximation $\Delta L / \lambda \ll f_{L}^{\prime 2} /\left(x^{2}+y^{2}\right)_{\max }$ is not valid, it can be shown by means of more strict analysis that under some additional assumptions an additional transversal phase modulation factor, $\exp \left[-2 \pi i \Delta L\left(x^{2}+y^{2}\right) /\left(\lambda f_{L}^{\prime 2}\right)\right]$, appears in the expression of the coherence function, $\Gamma_{L}(\omega ; x, y)$. If present, such transversal phase modulation of the coherence function can be corrected numerically.

\section{Numerical Correction of the Coherence Function}

\section{A. Temporal Spectrum Gate and Angular Spectrum Gate}

In a swept-source OCT/OCM experiment, the coherence function (11) for every particular quasimonochromatic state of the swept source can be reconstructed from the experimental interference patterns in a number of ways, including phase shifting reconstructions, off-axis reconstructions or Fourier filtration in the temporal spectrum domain [i.e., when a sufficient optical delay is introduced between the interferometer arms to produce high frequency modulation over $\omega$ of the $\Gamma_{L}(\omega ; x, y)$ signal and to allow for separation from other terms in Eq. (1)]. In this section we shall consider the properties of the complex coherence function without considering the way it has been reconstructed from the raw interferometric data.

It can be clearly seen from Eqs. () and (11), that the coherence function of the sample and reference fields in an interference microscope with quasimonochromatic partially spatially coherent illumination has the shape of the convolution of the sample reflection coefficient distribution of interest, $r_{S}$, with a certain impulse response function. It means that the shape of $r_{S}$ can be reconstructed by means of Fourier analysis. Let us consider the transversal spatial spectrum of $\Gamma_{L}$ :

$$
\tilde{\Gamma}_{L}\left(\omega ; k_{x}, k_{y}\right)=\iint \Gamma_{L}(\omega ; x, y) \exp \left[-i\left(k_{x} x+k_{y} y\right)\right] \mathrm{d} x \mathrm{~d} y .
$$

Then, we can obtain for the spatial spectrum of the coherence function when imaging the multilayered model sample,

$$
\begin{aligned}
& \tilde{\Gamma}_{L}\left(\omega ; k_{x}, k_{y}\right) \approx \mu_{0}(\omega) M^{2}\left[\prod_{j=1}^{N} t_{j-1, j}(\omega) t_{j, j-1}(\omega)\right] \\
& \times \tilde{r}_{S}\left(\omega ;-M k_{x},-M k_{y}\right) \Xi\left(\omega ; k_{x}, k_{y}\right), \\
& \Xi\left(\omega ; k_{x}, k_{y}\right) \\
& =\exp [2 i k \Delta L] \iint \mathrm{d} x_{S} \mathrm{~d} y_{S} \exp \left[-i M\left(k_{x} x_{S}+k_{y} y_{S}\right)\right] \\
& \times \iint A\left(\omega ; x_{3}, y_{3}\right) \exp \left[i k \sum_{j=0}^{N} \Delta z_{j} \sqrt{n_{j}^{2}-\frac{x_{3}^{2}+y_{3}^{2}}{f^{\prime 2}}}\right] \\
& \times \exp \left[-i \frac{k}{f^{\prime}}\left(x_{3} x_{S}+y_{3} y_{S}\right)\right] \mathrm{d} x_{3} \mathrm{~d} y_{3} \\
& \times \int A_{i}\left(\omega ; x_{0}, y_{0}\right) \exp \left\{i k \left[\left(z_{S}-2 z_{R}+|f|\right) \sqrt{n_{0}^{2}-\frac{x_{0}^{2}+y_{0}^{2}}{f^{\prime 2}}}\right.\right. \\
& \left.\left.+\sum_{j=1}^{N} \Delta z_{j} \sqrt{n_{j}^{2}-\frac{x_{0}^{2}+y_{0}^{2}}{f^{\prime 2}}}\right]\right\} \\
& \times \exp \left[-i \frac{k}{f^{\prime}}\left(x_{0} x_{S}+y_{0} y_{S}\right)\right] \mathrm{d} x_{0} \mathrm{~d} y_{0},
\end{aligned}
$$

$$
\begin{aligned}
& A_{i}\left(\omega ; x_{0}, y_{0}\right) \\
& =I_{0}\left(\omega ; x_{0}, y_{0}\right) A^{*}\left(\omega ;-x_{0},-y_{0}\right) r_{R}^{*}\left(\omega ;-k \frac{x_{0}}{f^{\prime}},-k \frac{y_{0}}{f^{\prime}}\right),
\end{aligned}
$$

where the tilde sign in $\tilde{r}_{S}$ (and everywhere in this paper) denotes the transversal spatial spectrum, similarly to Eq. (12) and $M=f_{L}^{\prime} / f^{\prime}$ is the absolute value of transversal image magnification. In Eq. (13), we have neglected the angular dependence of the transmission coefficients of the outer layers, $t_{j-1, j}$ and $t_{j, j-1}$. It can be seen from Eq. (13) that the $A_{i}\left(\omega ; x_{0}, y_{0}\right)$ function stands quite similarly to the aperture function, $A\left(\omega ; x_{3}, y_{3}\right)$. However, $A_{i}$ is determined by the properties of the illumination rather than the properties of the imaging system. Therefore, we shall call the $A_{i}$ function the "illumination aperture" function. It is interesting to note that, when $z_{R}=|f|$, the shape of the coherence signal [Eq. (13)] is very similar to the case of scanning confocal Fourier domain OCT/OCM [35]. However, the illumination aperture in Eq. (13b) is determined by the intensity, $I_{0}$, of the illumination field, while in the confocal modality it is determined by the amplitude of the illumination. In particular, this means that, unlike the confocal modality, the illumination aperture shape of the full-field modality is not sensitive to the objective aberrations (provided that the objectives are identical in both arms). Also, it can be noted that the sectioning effect due to the illumination aperture is dependent in Eq. (13b) on the reference mirror position, $z_{R}$. 
For more detailed analysis, the function $\Xi\left(\omega ; k_{x}, k_{y}\right)$ can be represented as a multiplication:

$$
\begin{gathered}
\Xi\left(\omega ; k_{x}, k_{y}\right)=\Xi_{t}(\omega) \Xi_{a}\left(\omega ; k_{x}, k_{y}\right), \\
\Xi_{t}(\omega)=\exp \left\{2 i k\left[\Delta L+n_{0}\left(z_{S}-z_{R}\right)+\sum_{j=1}^{N} n_{j} \Delta z_{j}\right]\right\}, \\
\Xi_{a}\left(\omega ; k_{x}, k_{y}\right)=\Xi\left(\omega ; k_{x}, k_{y}\right) \Xi_{t}^{*}(\omega)
\end{gathered}
$$

It can be seen from Eqs. (13)-(15) that the $\Xi$ function changes the shape of the coherence signal, $\tilde{\Gamma}_{L}$, making it differ from the function of interest, $\tilde{r}_{S}$. The $\Xi_{t}(\omega)$ function affects only the temporal spectrum, $\omega$ (and corresponds to the signal of conventional OCT with low NAs), while $\Xi_{a}\left(\omega ; k_{x}, k_{y}\right)$ affects predominantly the spectrum of the transversal spatial frequencies $\left(k_{x}, k_{y}\right)$, which is often regarded to as the angular spectrum. The effect of $\Xi_{t}(\omega)$ corresponds to longitudinal coherence gating due to the temporal spectrum (temporal spectrum gate), while the effect of $\Xi_{a}\left(\omega ; k_{x}, k_{y}\right)$ corresponds to the blurring due to defocus and longitudinal coherence gating due to the angular spectrum (angular spectrum gate). The limitations of this study do not allow us to study in detail the physical interpretation and terminology of these coherence effects. Investigation of the coherence effects due to the temporal and angular spectra of the optical field for different modalities of low-coherence interferometers can be found in the literature [15,24,26,27,29,33,34,36-38].

\section{B. Transversal Frequency Response Analysis and Numerical Processing Technique}

Let us consider the transversal spatial frequency response of the coherence signal, which is determined by the shape of the $\Xi_{a}\left(\omega ; k_{x}, k_{y}\right)$ function. The graphs in Fig. 2 represent the absolute value and the argument of the $\Xi_{a}\left(\omega ; k_{x}, k_{y}\right)$ function for the case when the sample consists of a single surface, $n_{0}=1$; $z_{R}=|f| ; \Delta z_{0}=\Delta z$; the imaging aperture function is circular, $\mathrm{NA}=0.1$. Figures $2(\mathrm{a})$ and 2(c) present the extreme case of point-like illumination aperture, i.e., when $A_{i}\left(\omega ; x_{0}, y_{0}\right) \approx A_{i}(\omega) \delta\left(x_{0}\right) \delta\left(y_{0}\right)$ and NAi $\rightarrow 0$. It corresponds to spatially transversally coherent illumination over the sample. Figures 2(b) and 2(d) present the case of relatively wide circular illumination aperture, with $\mathrm{NAi}=0.05$, which corresponds to low spatial coherence illumination of the sample. Owing to the revolution symmetry of the illumination and imaging systems, the $\Xi_{a}\left(\omega ; k_{x}, k_{y}\right)$ function has circular symmetry, and hence $\Xi_{a}\left(\omega ; k_{x}, 0\right)$ presented in Fig. 2 has the same shape as $\Xi_{a}\left(\omega ; 0, k_{y}\right)$. Computation of theoretical expressions and numerical processing of experimental data, presented in Figs. $\underline{2}-\underline{5}$, have been performed using
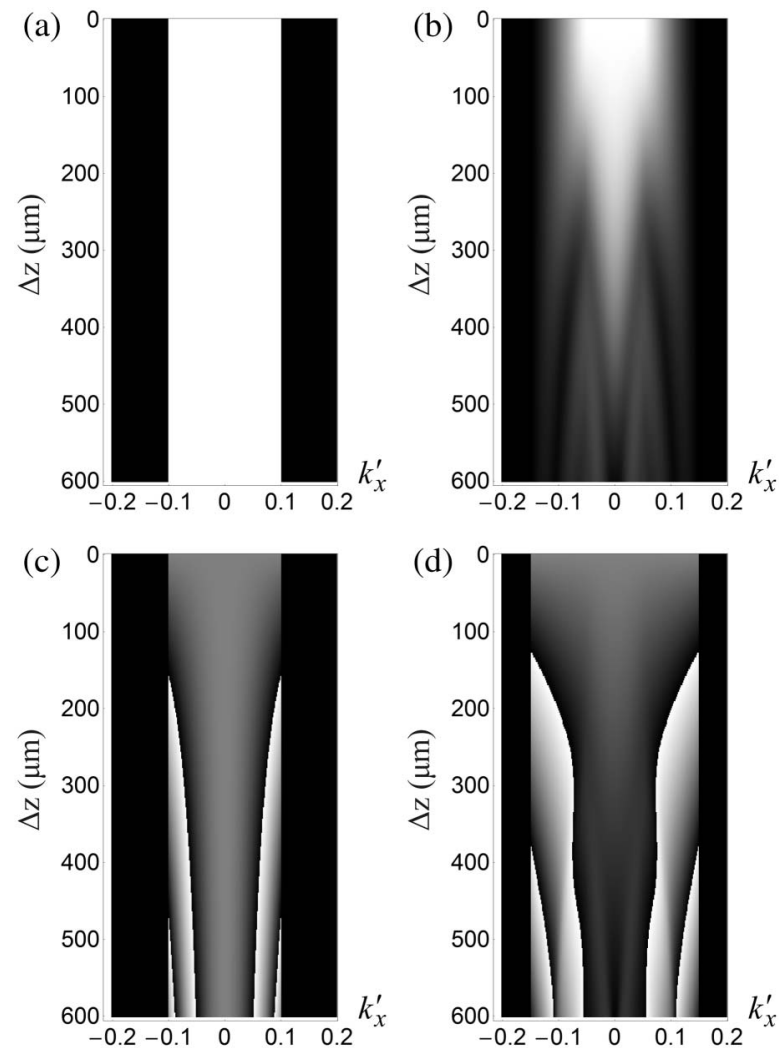

Fig. 2. Absolute value (a), (b) and phase (c), (d) of the $\Xi_{a}\left(\omega ; k_{x}, 0\right)$ distribution versus the reduced spatial frequency $k_{x}^{\prime}$ and the defocus value $\Delta z$; (a), (c), $\mathrm{NA}=0.1, \mathrm{NAi} \rightarrow 0$; (b), (d), NA =0.1, $\mathrm{NAi}=0.05$. A frequency $\omega=1.2 \times 10^{15} \mathrm{~Hz}(\lambda=1.57 \mu \mathrm{m})$ was considered. Note that we introduced here the normalized spatial frequency $k_{x}^{\prime}=M \times k_{x} / k$ where $M$ is the magnification of the imaging system.

home-made programs, written in Wolfram Mathematica software.

As can be seen in Fig. 2 , the modulation of coherence signal $\tilde{\Gamma}_{L}$ by $\Xi_{a}$ affects both its phase and amplitude. A straightforward approach to numerical correction of the experimental data and reconstruction of a sharp image of the interface of interest even outside the focus depth would be the following: for every particular frequency $\omega$, divide $\tilde{\Gamma}_{L}\left(\omega ; k_{x}, k_{y}\right)$ by appropriate $\Xi\left(\omega ; k_{x}, k_{y}\right)$ function. This can be realized for nonzero $\Xi$ values, if the signalto-noise ratio (SNR) of the experimental data $\Gamma_{L}$ is high enough. Otherwise, such direct division would also lead to an increase of the noise.

To avoid problems with SNR, a more reliable processing algorithm can be implemented, which consists in multiplication of $\tilde{\Gamma}_{L}$ by a special correction function. To produce sharp, coherence gated image of an interface, located under $N$ sample layers, the $\tilde{\Gamma}_{L}$ function should be multiplied by

$$
\begin{aligned}
& \Psi\left(\omega ; k_{x}, k_{y}\right) \\
& \quad= \begin{cases}\Xi^{*}\left(\omega ; k_{x}, k_{y}\right) /\left|\Xi\left(\omega ; k_{x}, k_{y}\right)\right|, & \text { if }\left|\Xi\left(\omega ; k_{x}, k_{y}\right)\right|>0, \\
0, & \text { if }\left|\Xi\left(\omega ; k_{x}, k_{y}\right)\right|=0 .\end{cases}
\end{aligned}
$$



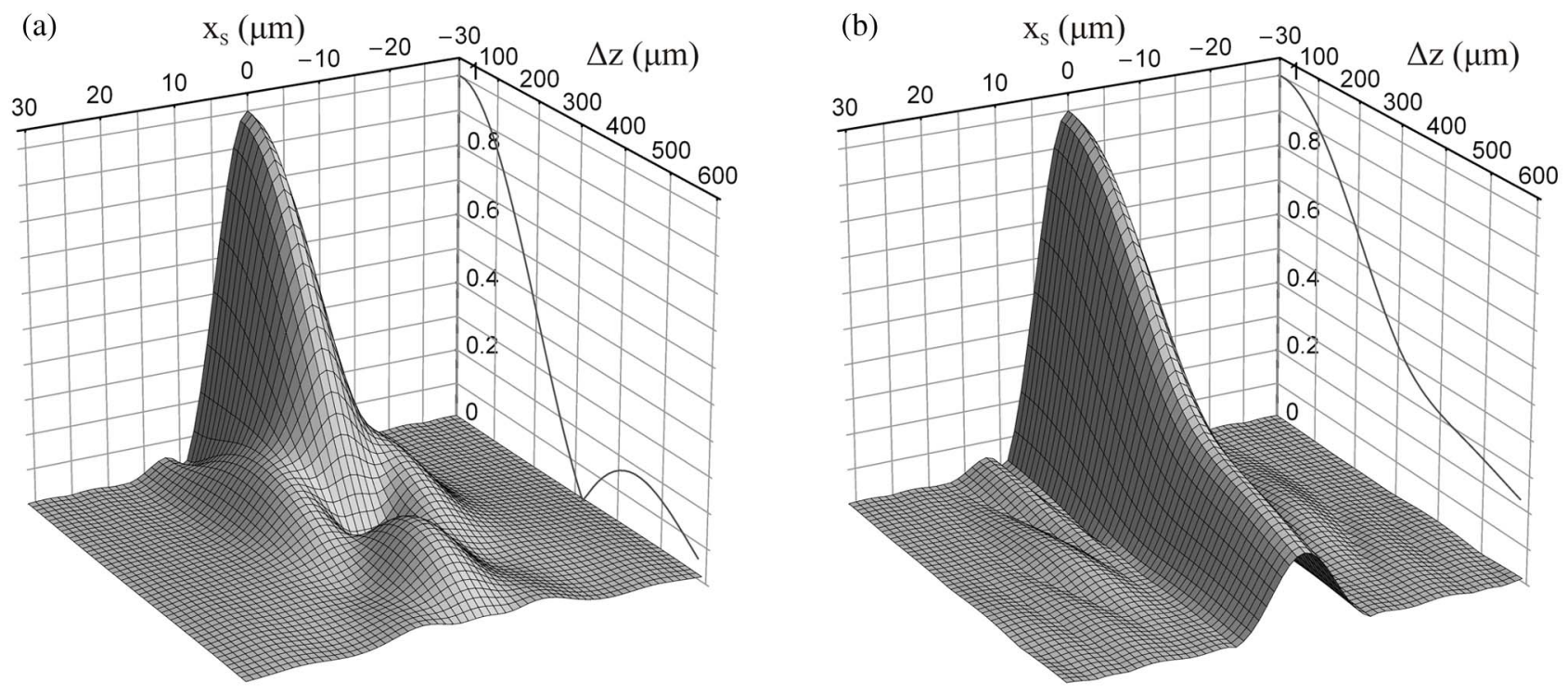

(c)

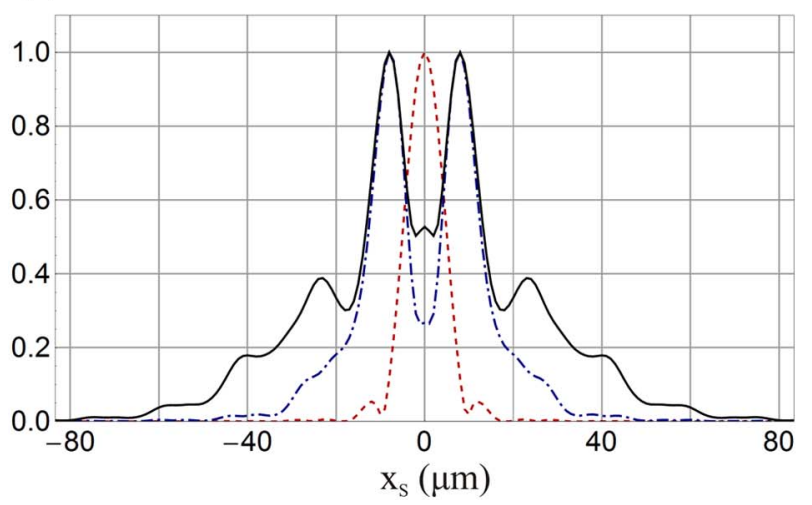

(d)



Fig. 3. (a) and (b) $3 \mathrm{D}$ graph represents the absolute value of the $\Gamma_{\mathrm{PSF}}\left(\omega ; x_{S}, 0\right)$ distribution over $x_{S}$ versus defocus value $\Delta z$; the $2 \mathrm{D}$ projected plot corresponds to the evolution of the absolute value of $\Gamma_{\mathrm{PSF}}(\omega ; 0,0)$ with respect to the defocus value. (a), without numerical correction; (b), with numerical correction according to Eq. (16a). (c) and (d) absolute value of the $\Gamma_{\mathrm{PSF}}\left(\omega ; x_{S}, 0\right)$ distribution over $x_{S}$ after normalization to unity maximum for comparison of the widths for three defocus values: $\Delta z=0 \mu \mathrm{m}$ (red dash line), $\Delta z=300 \mu \mathrm{m}$ (blue point-dash line), $\Delta z=600 \mu \mathrm{m}$ (black continuous line), (c), without numerical correction; (d), with numerical correction according to Eq. (16a). For all images NA $=0.1, \mathrm{NAi}=0.05, \omega=1.2 \times 10^{15} \mathrm{~Hz}$.

The resultant function $\tilde{\Gamma}_{L}\left(\omega ; k_{x}, k_{y}\right) \Psi\left(\omega ; k_{x}, k_{y}\right)$ should then be integrated (summed) over $\omega$ and Fourier transformed over $\left(k_{x}, k_{y}\right)$.

$\Psi\left(\omega ; k_{x}, k_{y}\right)$ is only a phase function with unity absolute value; its zero part is necessary just to avoid division by zero and suppress some noise. This means that multiplication of $\tilde{\Gamma}_{L}$ by $\Psi$ does not affect the SNR of $\tilde{\Gamma}_{L}$ data, except for suppression of some high-frequency noise, and leads to image sharpening or "numerical focusing".

It can be seen in Fig. 2(b), that the amplitude of the $\Xi_{a}$ function decreases with increase of the defocus distance. The larger the illumination aperture, the stronger this effect of $\Xi_{a}$ amplitude decrease. It follows readily from Eq. (13) that if the shape of illumination intensity distribution $I_{0}$ becomes point-like, i.e., when $A_{i}\left(\omega ; x_{0}, y_{0}\right) \approx A_{i}(\omega) \delta\left(x_{0}\right) \delta\left(y_{0}\right)$, then the shape of the coherence function (13) becomes similar to the case of interference microscopy with transversally coherent plane-wave illumination
[19], which does not have a numerical refocusing range limitation caused by the SNR [Fig. 2(a)]. It means that if the illumination aperture is narrow and the numerical refocusing distance is not too large, the numerical processing algorithm for spatially coherent illumination [19] can be applied to FF-SSOCM with partial spatial coherence of illumination. The use of this algorithm provides certain advantage in computation speed in comparison to Eq. (16a). However, for larger illumination aperture sizes, the shape of phase modulation $\Psi\left(\omega ; k_{x}, k_{y}\right)$ becomes different, which can be seen from comparison of Figs. 2(c) and 2(d). Hence, in general, the numerical processing according to Eq. (16a) should be used for producing sharp coherence gated FF-SSOCM images with partially transversally coherent illumination. For the sake of improvement of the computation speed, a C-mode-like processing algorithm can be applied. Then the $\tilde{\Gamma}_{L}\left(\omega ; k_{x}, k_{y}\right)$ function should be multiplied by 
(a)

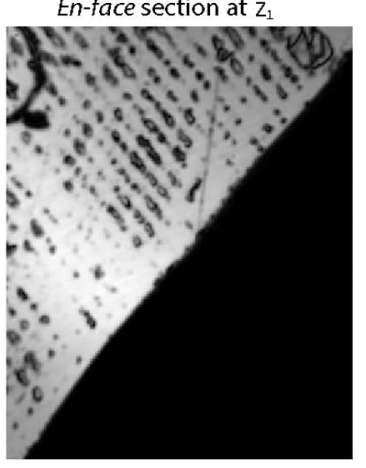

En-face section at $\mathrm{Z}_{2}$

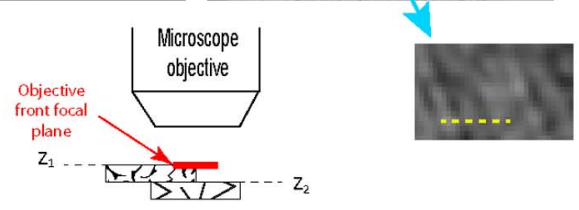

En-face section at $z_{1}$

(c)

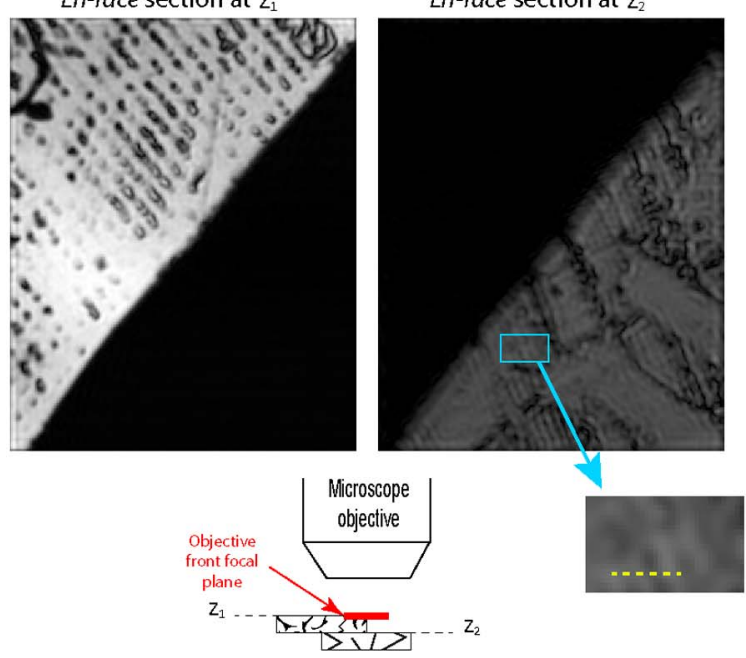

En-face section at $z_{1}$

(b)
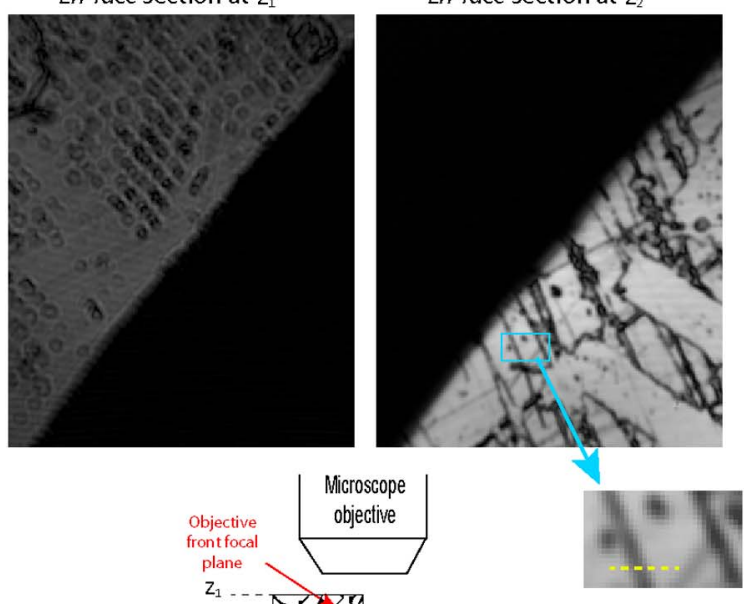

En-face section at $\mathrm{z}_{1}$

(d)

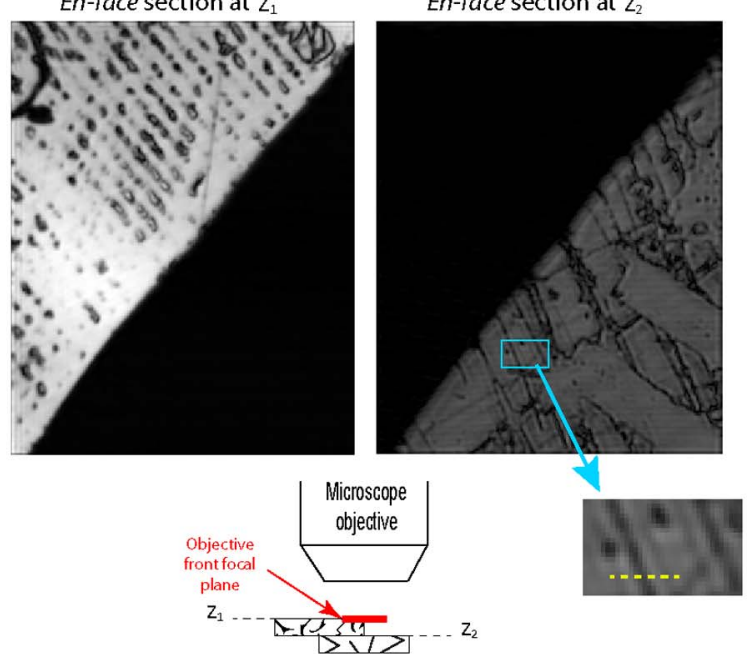

Fig. 4. Comparison of FF-SSOCM images obtained without the focalization algorithm for two mechanically focused position (a), (b) and with numerical focalization (c), (d) by using the algorithm for spatially coherent illumination (c) or the proposed algorithm (d). Positions of cuts (see Fig. $\underline{5}$ ) along the $x_{S}$ axis are indicated in the zoomed windows. The field of view is $700 \mu \mathrm{m} \times 870 \mu \mathrm{m}$.

$\Psi_{a}\left(\omega ; k_{x}, k_{y}\right)$
$= \begin{cases}\Xi_{a}^{*}\left(\omega ; k_{x}, k_{y}\right) /\left|\Xi_{a}\left(\omega ; k_{x}, k_{y}\right)\right|, & \text { if }\left|\Xi_{a}\left(\omega ; k_{x}, k_{y}\right)\right|>0, \\ 0, & \text { if }\left|\Xi_{a}\left(\omega ; k_{x}, k_{y}\right)\right|=0,\end{cases}$

and the resultant function $\tilde{\Gamma}_{L}\left(\omega ; k_{x}, k_{y}\right) \Psi_{a}\left(\omega ; k_{x}, k_{y}\right)$ should then be Fourier transformed over the three frequencies $\left(\omega ; k_{x}, k_{y}\right)$. Such numerical focus adjustment is performed for several depths inside the sample, with a step of the order of the focus depth (not at the depth sampling step as in Eq. (16a) and the obtained data are combined into a final 3D image. In this case, the required number of numerical focus adjustments is decreased similarly to decrease the number of optical focus adjustments in the mechanical C-mode scanning approach [39], and provides higher numerical processing speed.

As can be seen from Eqs. (13)-(15), for every frequency $\omega$, a $2 \mathrm{D}$ Fourier transform of the $\Xi_{a}$ function over the spatial frequencies $\left(k_{x}, k_{y}\right)$ determines the transversal impulse response (pointspread-function, PSF) of the coherence function $\Gamma_{L}(\omega ; x, y)$. Scaled to the object space coordinates, this function can be denoted as $\Gamma_{\mathrm{PSF}}(\omega ; x / M, y / M)$. Fig. 3 illustrates the influence of the numerical correction according to Eq. (16a) on the transversal PSF of the coherence function in the case of relatively wide illumination aperture $\mathrm{NAi}=0.05$. Owing to the revolution symmetry of the illumination and imaging systems, the $\Gamma_{\mathrm{PSF}}\left(\omega ; x_{S}, y_{S}\right)$ function has circular symmetry, so that $\Gamma_{\mathrm{PSF}}\left(\omega ; x_{S}, 0\right)$ presented in Fig. 3 has the same shape as $\Gamma_{\mathrm{PSF}}\left(\omega ; 0, y_{S}\right)$. The processing according to Eq. (16a) preserves a sharp PSF in spite of the defocus, as can be seen from Fig. 3(b). This effect can be even more clearly seen by comparison of Figs. 3(c) and 3(d), which presents the PSFs for three values of defocus $(0,300$, and $600 \mu \mathrm{m})$, after normalization to unity maximum at each defocus position.

Since the illumination aperture is relatively wide, the amplitude is lower in the defocus region. 
This amplitude decrease can be clearly seen from the two graphs in Fig. 3(b). For example, at $600 \mu \mathrm{m}$ from the focus plane the maximum value of the $\Gamma_{\mathrm{PSF}}$, even after numerical correction, is reduced by a factor of 5 compared to the maximum value of the focused $\Gamma_{\mathrm{PSF}}$.

As can be seen in Fig. 2(b), wider illumination aperture results in wider nonzero regions of $\left|\Xi_{a}\left(\omega ; k_{x}, k_{y}\right)\right|$, which enables the detection of larger spatial frequencies. Also, for wider illumination aperture the decrease of $\left|\Xi_{a}\left(\omega ; k_{x}, k_{y}\right)\right|$ with increase of $k_{x}$ and $k_{y}$ becomes smoother, which leads to suppression of sidelobes in the PSF.

Finally, it should be noted that when the complex coherence signal reconstruction is based on Fourier filtration in the temporal frequency domain, the numerical processing procedure, according to Eq. (16), can be applied directly to the interference signal without prior reconstruction of the complex coherence function. This results from the linearity of this processing. Therefore, the presence of other interference terms [see Eq. (1)] does not affect the correction of the complex coherence function.

\section{Experimental Verification of the Numerical Processing Technique}

\section{A. Experimental Setup}

To verify the applicability of our numerical processing technique to focus correction, we have built a dedicated FF-SSOCM system. The experimental set-up is based on a Linnik-type interferometer, as in conventional full-field OCT/OCM [15]. A schematic of the experimental arrangement is shown in Fig. 1. Two identical dry microscope objectives are used $\overline{(5} \times, 0.1$ N.A.). The light source is a swept-source laser (Model AQ4320d, Ando Electric Co.) emitting light continuously from $\nu_{1}=1.97 \times 10^{14} \mathrm{~Hz}\left(\lambda_{1}=1.52 \mu \mathrm{m}\right)$ to $\nu_{2}=$ $1.85 \times 10^{14} \mathrm{~Hz}\left(\lambda_{2}=1.62 \mu \mathrm{m}\right)$ with an output optical power of $5 \mathrm{~mW}$. The light emitted by the laser is directed into a single-mode fiber and then collimated by a fiber collimation package (F280 APC-C, Thorlabs). A rotating diffuser, conjugated with the aperture plane of the microscope objectives, is used to reduce the spatial coherence of the illumination and then remove the coherent noise related to it. Unlike conventional full-field OCM, the reference mirror is immobile, since no phase shifting is used here to extract the interferometric signal amplitude and no mechanical scan is required to acquire the depth information in the sample. Furthermore, the transverse information is acquired in parallel by an area camera, leading to a full 3D motion-free imaging system. The images are projected onto an Indium Gallium Arsenide (InGaAs) camera (OWL SW1.7-CL-HS, RaptorPhotonics, $320 \times 256$ pixels, 14 bits) via an achromatic $400 \mathrm{~mm}$ lens optimized in the $1.5 \mu \mathrm{m}$ wavelength region. The field of view is $700 \mu \mathrm{m} \times 870 \mu \mathrm{m}$.

\section{B. Methods}

The source is swept in wavelength to acquire the spectral interference pattern which is then processed in order to obtain directly en face tomographic images of the sample owing to the full field illumination. To produce a 3D-image, $2562 \mathrm{D}$ interference patterns are acquired and then processed after applying a Gaussian apodization. A sufficient optical delay, $2 \Delta L$, was introduced between the interference microscope arms prior to the imaging, to allow sufficient separation of the complex coherence term $\Gamma_{L}(\omega ; x, y)$ from the other terms of Eq. (1). Since the swept-source laser generates light that is nonlinearly sampled in wavenumber, a numerical pixel interpolation is done to provide equidistant sampling [40]. Each of the 256 interference patterns corresponds to a source linewidth of $3.91 \times 10^{-1} \mathrm{~nm}$ $\left(47.6 \times 10^{9} \mathrm{~Hz}\right)$, which provides a theoretical maximum imaging depth in the sample of $1580 \mu \mathrm{m}$ [41]. Besides, before applying the numerical focusing algorithms, for every $\omega$ the spatial spectrum of coherence function is centered by appropriate linear phase modulation over $(x, y)$, correcting the effect of some residual misadjustment in the setup

The imaging depth of field is governed by the NA of the microscope objectives and the mean temporal frequency. In our experimental conditions, the depth of field is around $z_{\max }=150 \mu \mathrm{m}$, and corresponds to the actual imaging depth without focalization correction.

By using the proposed numerical processing according to Eq. (16a) (which is applied directly to the interference signal, as described above in Subsection 3.B), this yields numerically focused images and expands the imaging depth which depends then mostly on the illumination properties [Fig. 3(b)].

The transverse resolution is also related to the numerical aperture of the microscope objectives (NA) and of the illumination (NAi), and to the mean temporal frequency $(\omega)$. The theoretical full width at half-maximum of the transversal PSF was calculated to be $\sim 10 \mu \mathrm{m}$ (with $\mathrm{NA}=0.1, \mathrm{NAi}=0.05$ and $\omega=1.2 \times 10^{15} \mathrm{~Hz}$ ). In the same conditions, the width of the step-response [42] has a theoretical value of $\sim 6 \mu \mathrm{m}$. Experimentally, the width of the step-response was estimated to be $\sim 8 \mu \mathrm{m}$. The deviation of the theoretical and experimental values is presumably caused by the objectives, which are not optimized for these wavelengths.

The longitudinal resolution, depending on the temporal coherence length of the light source, was measured, after apodization of the spectral interference pattern, to be $21 \mu \mathrm{m}$ in air.

The use of such microscope objectives, with relatively high numerical aperture $(\mathrm{NA}=0.1)$, yields better transverse resolution than in conventional swept-source OCT (SSOCT) at the cost of the depth of field, which is then much smaller than the usual one in SSOCT. However, the numerical focalization expands the maximum reachable imaging depth and makes it similar to the one of conventional SSOCT.

By considering low spatial coherence illumination, the larger the illumination numerical aperture, the 
lower the amplitude of the detected signal from defocused regions in the sample. Assuming that the illumination aperture function has a uniform circular shape, we have estimated the illumination numerical aperture (NAi) to be 0.05 . Then, the NAi limits the imaging depth, since, without enough intensity, no image can be produced, even if they are numerically focused.

Given the evolution of the PSF amplitude along the longitudinal axis after numerical correction [Figs. 3(b) and 3(d)], the use of an illumination aper-

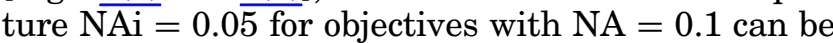
considered as a good trade-off between transverse resolution and loss of signal from defocused regions.

\section{Results}

For testing the performance of our numerical processing technique, we have compared tomographic images obtained with our FF-SSOCM technique without and with focalization algorithms. We choose a sample whose longitudinal extent exceeds the optical depth of field. The sample consists of two pieces of an integrated circuit wafer placed one on the other. The two pieces partially overlap making the sample represent two highly contrasted structures located in two distinct parallel planes. The difference of height between the two planes is $\sim 330 \mu \mathrm{m}$.

The different images are presented in Fig. 4, where the upper and lower circuits appear on the left and right parts of the images respectively. Two sets of figures are displayed. Images in Figs. 4(a) and 4(b) correspond to FF-SSOCM processing without numerical focalization. Images in Figs. 4(c) and 4(d) compare the two focalization algorithms. As indicated on the figure, in Fig. 4(b) the front focal plane of the microscope objective matches with the lower circuit whereas all of the three other images correspond to a mechanically focused position on the upper circuit. Figure 4(c) presents the result of processing with the algorithm proposed in [19] for spatially coherent illumination, and Fig. 4(d) presents the result after using our specific algorithm. It can be seen that, without the focalization algorithm [Figs. 4(a) and 4(b)], only the images of the mechanically focused surfaces appear sharp. However, application of an appropriate focalization numerical processing [Fig. 4(d)] allows us to obtain sharp images of the two circuits irrespective of their axial positions. In accordance with the theoretical analysis presented in Subsection 3.B, the amplitude of the defocused image becomes lower in comparison to the focused one because of the wide illumination aperture. Figure 4(c) shows that the algorithm for interference microscopes with planewave illumination is not suitable to numerically focus with wide illumination aperture.

Profiles of the absolute value of the coherence function along a line (see yellow-dashed line in Fig. 4) across a thin scratch on the sample surface are presented in Fig. 5 for quantitative illustration of the effect of numerical focalization. It can be seen from Fig. 5 that the sharp scratch profile visible in the

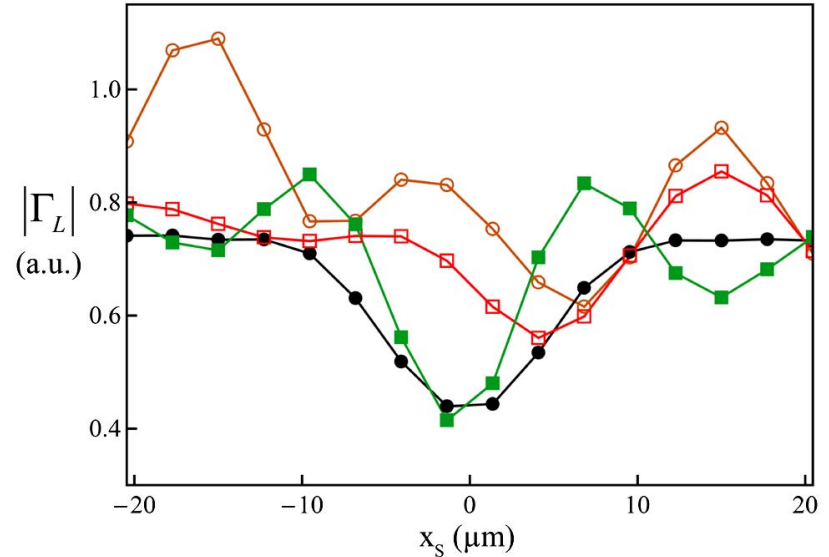

Fig. 5. Distributions of the absolute value of the reconstructed coherence function along the $x_{S}$ axis in the lower circuit images (see Fig. 4) with mechanical focalization on the lower circuit (black line with filled dots) and mechanical focalization on the upper circuit (all other data points): without numerical focusing (orange line with white dots), with numerical focusing for spatially coherent illumination (red line with white rectangles) and with numerical focusing by the proposed technique (green line with filled rectangles). The distributions for the case of focalization on the upper circuit are magnified 4 times in comparison to the distributions for focalization on the lower circuit. Unlike the algorithm for spatially coherent illumination, the proposed algorithm for arbitrary state of spatial coherence clearly reveals the dark thin scratch, visible in the optically focused image.

focused image (black line with filled dots) gets blurred in the defocused image whether the numerical focusing for spatially coherent illumination is applied or not. However, our specific algorithm for partially spatially coherent illumination applied to the defocused OCM data (green line with filled rectangles) is able to restore a sharp profile similar to the one from the optically focused image, except for the presence of side lobes. We suppose that this deviation is caused by some artifacts in the numerical processing, in first place by the ignorance of the actual illumination aperture shape which is supposed to have a uniform circular shape. Enhancement of the image quality could be achieved by complete characterization of the illumination aperture shape.

\section{Conclusions}

We have demonstrated a 3D imaging technique, based on the combination of FF-SSOCM with low spatial coherence illumination and an original numerical processing technique for extension of the imaging depth of field. It is shown, theoretically and experimentally, that this numerical focusing enhances the sharpness and contrast of the reconstructed images.

This numerical processing includes the processing for spatially coherent plane-wave illumination [19] as a particular case of extremely narrow illumination aperture. This effect can be explained using the terminology of transversal coherence of illumination 
field, incident on the sample. The smaller transversal coherence length of this field corresponds to wider illumination aperture and vice versa. Hence, the case of narrow illumination aperture corresponds to transversally almost coherent illumination, similarly to plane-wave illumination. The proposed numerical processing is more general and allows for numerical focusing even in the case of low spatial coherence illumination.

As can be seen from the numerical simulations and experimental results, the use of a wide illumination aperture leads to a decrease of the coherence signal amplitude with increased defocus. If this decrease of the amplitude is tolerable through the whole required image depth, the system is completely motion free and does not require mechanical displacement of the optical elements or the sample. If this amplitude decrease is too large, a smaller illumination aperture should be chosen or some longitudinal scanning can still be performed in the C-mode regime [39]. In the latter case, the proposed technique provides an extension of the original depth of field and decrease of the number of required mechanical adjustments.

On the other hand, it should be noted that a very high sensitivity is peculiar to OCT/OCM systems. This sensitivity is necessary to overcome the signal attenuation due to scattering and absorption and detect the weak signals from structures inside the imaged sample. It means that if the optical focus is adjusted at the maximum required image depth, the effects of signal attenuation due to scattering and absorption inside the sample and attenuation due to defocus would somewhat compensate each other, leading to more uniform SNR in the imaged volume. The proposed FF-SSOCM system could then be used as a totally motion free imaging system with extended imaging depth, even with wider numerical apertures. The experimental investigation of the peculiarities of imaging scattering samples should be a topic of further research.

We have considered here the possibilities of the technique for tomographic 3D imaging. The technique can also be considered as a reflection-mode digital holographic microscope (DHM) with partially spatially coherent illumination for coherent-noisefree quantitative phase imaging. All the theoretical results derived in this paper can be directly applied to this DHM mode as well as to the discussed FF-SSOCM mode.

The authors are grateful to Nicolas Dubreuil for lending the swept laser source. A. Grebenyuk and V. Ryabukho have been supported in part by Russian Federation state contract 14.B37.21.0728 and grant NSh-1177.2012.2. A. Grebenyuk is also grateful to the Development Program of National Research Saratov State University for supporting his stay at the Institut d'Optique Graduate School, where the experimental investigations were carried out.

\section{References}

1. D. Huang, E. A. Swanson, C. P. Lin, J. S. Schuman, W. G. Stinson, W. Chang, M. R. Hee, T. Flotte, K. Gregory, C. A. Puliafito, and J. G. Fujimoto, "Optical coherence tomography," Science 254, 1178-1181 (1991).

2. W. Drexler and J. G. Fujimoto, eds. Optical Coherence Tomography (Springer, 2008).

3. F. Lexer, C. K. Hitzenberger, W. Drexler, S. Molebny, H. Sattmann, M. Sticker, and A. F. Fercher, "Dynamic coherent focus OCT with depth-independent transversal resolution," J. Mod. Opt. 46, 541-553 (1999).

4. Y. Wang, Y. Zhao, J. S. Nelson, Z. Chen, and R. S. Windeler, "Ultrahigh-resolution optical coherence tomography by broadband continuum generation from a photonic crystal fiber," Opt. Lett. 28, 182-184 (2003).

5. J. P. Rolland, P. Meemon, S. Murali, K. P. Thompson, and K. S. Lee, "Gabor-based fusion technique for optical coherence microscopy," Opt. Express 18, 3632-3642 (2010).

6. Z. Ding, H. Ren, Y. Zhao, J. S. Nelson, and Z. Chen, "Highresolution optical coherence tomography over a large depth range with an axicon lens," Opt. Lett. 27, 243-245 (2002).

7. R. A. Leitgeb, M. Villiger, A. H. Bachmann, L. Steinmann, and T. Lasser, "Extended focus depth for Fourier domain optical coherence microscopy," Opt. Lett. 31, 2450-2452 (2006).

8. T. S. Ralston, D. L. Marks, P. S. Carney, and S. A. Boppart, "Interferometric synthetic aperture microscopy," Nat. Phys. 3, 129-134 (2007).

9. D. K. Hamilton and C. J. R. Sheppard, "A confocal interference microscope," Optica Acta 29, 1573-1577 (1982).

10. J. A. Izatt, M. R. Hee, G. M. Owen, E. A. Swanson, and J. G. Fujimoto, "Optical coherence microscopy in scattering media," Opt. Lett. 19, 590-592 (1994).

11. L. F. Yu and M. K. Kim, "Wavelength-scanning digital interference holography for tomographic three-dimensional imaging by use of the angular spectrum method," Opt. Lett. 30, 2092-2094 (2005).

12. F. Montfort, T. Colomb, F. Charriere, J. Kuhn, P. Marquet, E. Cuche, S. Herminjard, and C. Depeursinge, "Submicrometer optical tomography by multiple-wavelength digital holographic microscopy," Appl. Opt. 45, 8209-8217 (2006).

13. D. Hillmann, C. Luhrs, T. Bonin, P. Koch, and G. Huttmann, "Holoscopy-holographic optical coherence tomography," Opt. Lett. 36, 2390-2392 (2011).

14. E. Beaurepaire, A. C. Boccara, M. Lebec, L. Blanchot, and H. Saint-Jalmes, "Full-field optical coherence microscopy," Opt. Lett. 23, 244-246 (1998).

15. A. Dubois, L. Vabre, A. C. Boccara, and E. Beaurepaire, "High-resolution full-field optical coherence tomography with a Linnik microscope," Appl. Opt. 41, 805-812 (2002).

16. L. Vabre, A. Dubois, and A. C. Boccara, "Thermal-light fullfield optical coherence tomography," Opt. Lett. 27, 530-532 (2002).

17. B. Laude, A. De Martino, B. Drévillon, L. Benattar, and L. Schwartz, "Full-field optical coherence tomography with thermal light," Appl. Opt. 41, 6637-6645 (2002).

18. D. L. Marks, T. S. Ralston, S. A. Boppart, and P. S. Carney, "Inverse scattering for frequency-scanned full-field optical coherence tomography," J. Opt. Soc. Am. A 24, 1034-1041 (2007).

19. A. A. Grebenyuk and V. P. Ryabukho, "Numerical correction of coherence gate in full-field swept-source interference microscopy," Opt. Lett. 37, 2529-2531 (2012).

20. B. Karamata, M. Leutenegger, M. Laubscher, S. Bourquin, T. Lasser, and P. Lambelet, "Multiple scattering in optical coherence tomography. II. Experimental and theoretical investigation of cross talk in wide-field optical coherence tomography," J. Opt. Soc. Am. A 22, 1380-1388 (2005).

21. D. Sacchet, M. Brzezinski, J. Moreau, P. Georges, and A. Dubois, "Motion artifact suppression in full-field optical coherence tomography," Appl. Opt. 49, 1480-1488 (2010).

22. B. Povazay, A. Unterhuber, B. Hermann, H. Sattmann, H. Arthaber, and W. Drexler, "Full-field time-encoded frequencydomain optical coherence tomography," Opt. Express 14, 7661-7669 (2006). 
23. A. A. Grebenyuk and V. P. Ryabukho, "Theoretical model of volumetric objects imaging in a microscope," Proc. SPIE 8430, 84301B (2012).

24. A. A. Grebenyuk and V. P. Ryabukho, "Coherence effects of thick objects imaging in interference microscopy," Proc. SPIE 8427, 84271M (2012).

25. L. Mandel and E. Wolf, Optical Coherence and Quantum Optics (Cambridge University, 1995), Section 2.4.

26. P. de Groot and X. C. de Lega, "Signal modeling for lowcoherence height-scanning interference microscopy," Appl. Opt. 43, 4821-4830 (2004).

27. V. Ryabukho, D. Lyakin, and M. Lobachev, "Longitudinal pure spatial coherence of a light field with wide frequency and angular spectra," Opt. Lett. 30, 224-226 (2005).

28. A. Dubois, G. Moneron, and C. Boccara, "Thermal-light fullfield optical coherence tomography in the $1.2 \mu \mathrm{m}$ wavelength region," Opt. Commun. 266, 738-743 (2006).

29. A. Safrani and I. Abdulhalim, "Spatial coherence effect on layer thickness determination in narrowband full-field optical coherence tomography," Appl. Opt. 50, 3021-3027 (2011).

30. T. S. Ralston, D. L. Marks, P. S. Carney, and S. A. Boppart, "Inverse scattering for optical coherence tomography," J. Opt. Soc. Am. A 23, 1027-1037 (2006).

31. A. A. Grebenyuk and V. P. Ryabukho, "Numerical reconstruction of volumetric image in swept-source interference microscopy," AIP Conf. Proc. 1537, 147-154 (2013).

32. M. D. Modell, V. Ryabukho, D. Lyakin, V. Lychagov, E. Vitkin, I. Itzkan, and L. T. Perelman, "Autocorrelation low coherence interferometry," Opt. Commun. 281, 1991-1996 (2008).

33. J. Rosen and A. Yariv, "Longitudinal partial coherence of optical radiation," Opt. Commun. 117, 8-12 (1995).
34. V. Ryabukho, D. Lyakin, and M. Lobachev, "Influence of longitudinal spatial coherence on the signal of a scanning interferometer," Opt. Lett. 29, 667-669 (2004).

35. A. A. Grebenyuk and V. P. Ryabukho, "Numerical reconstruction of 3D image in Fourier domain confocal optical coherence microscopy," in Proceedings of the International Conference on Advanced Laser Technologies (ALT) (Bern Open, 2013), pp. 1-5.

36. I. Zeylikovich, "Short coherence length produced by a spatial incoherent source applied for the Linnik-type interferometer," Appl. Opt. 47, 2171-2177 (2008).

37. I. Abdulhalim, "Competence between spatial and temporal coherence in full field optical coherence tomography and interference microscopy," J. Opt. A 8, 952-958 (2006).

38. V. P. Ryabukho, D. V. Lyakin, A. A. Grebenyuk, and S. S. Klykov, "Wiener-Khintchin theorem for spatial coherence of optical wave field," J. Opt. 15, 025405 (2013).

39. A. D. Aguirre and J. G. Fujimoto, "Optical coherence microscopy," in Optical Coherence Tomography, W. Drexler and J. G. Fujimoto, eds. (Springer, 2008), pp. 505-542.

40. M. D. Bayleyegn, H. Makhlouf, C. Crotti, K. Plamann, and A Dubois, "Ultrahigh resolution spectral-domain optical coherence tomography at $1.3 \mu \mathrm{m}$ using a broadband superluminescent diode light source," Opt. Commun. 285, 5564-5569 (2012).

41. M. A. Choma, M. V. Sarunic, C. H. Yang, and J. A. Izatt, "Sensitivity advantage of swept source and Fourier domain optical coherence tomography," Opt. Express 11, 2183-2189 (2003).

42. D. Sacchet, J. Moreau, P. Georges, and A. Dubois, "Simultaneous dual-band ultra-high resolution full-field optical coherence tomography," Opt. Express 16, 19434-19446 (2008). 